\title{
A case of severe hypoaldosteronism following unilateral adrenalectomy for Conn's Syndrome
}

\author{
Alistair Connell, Mark Cohen \\ Barnet and Chase Farm Hospitals NHS Trust
}

\section{Introduction}

A 58 year old female presented with a history of resistant hypertension and hypokalaemia, with normal renal function. Investigations confirmed primary hyperaldosteronism that was not suppressed following a standard saline infusion test. CT scanning revealed a $1.3 \mathrm{~cm}$ right-sided adrenal mass (fig.1).

Adrenal vein sampling confirmed right-sided unilateral hypersecretion of aldosterone; she underwent an uneventful right adrenalectomy, following which her BP was controlled on atenolol alone (fig. 2).

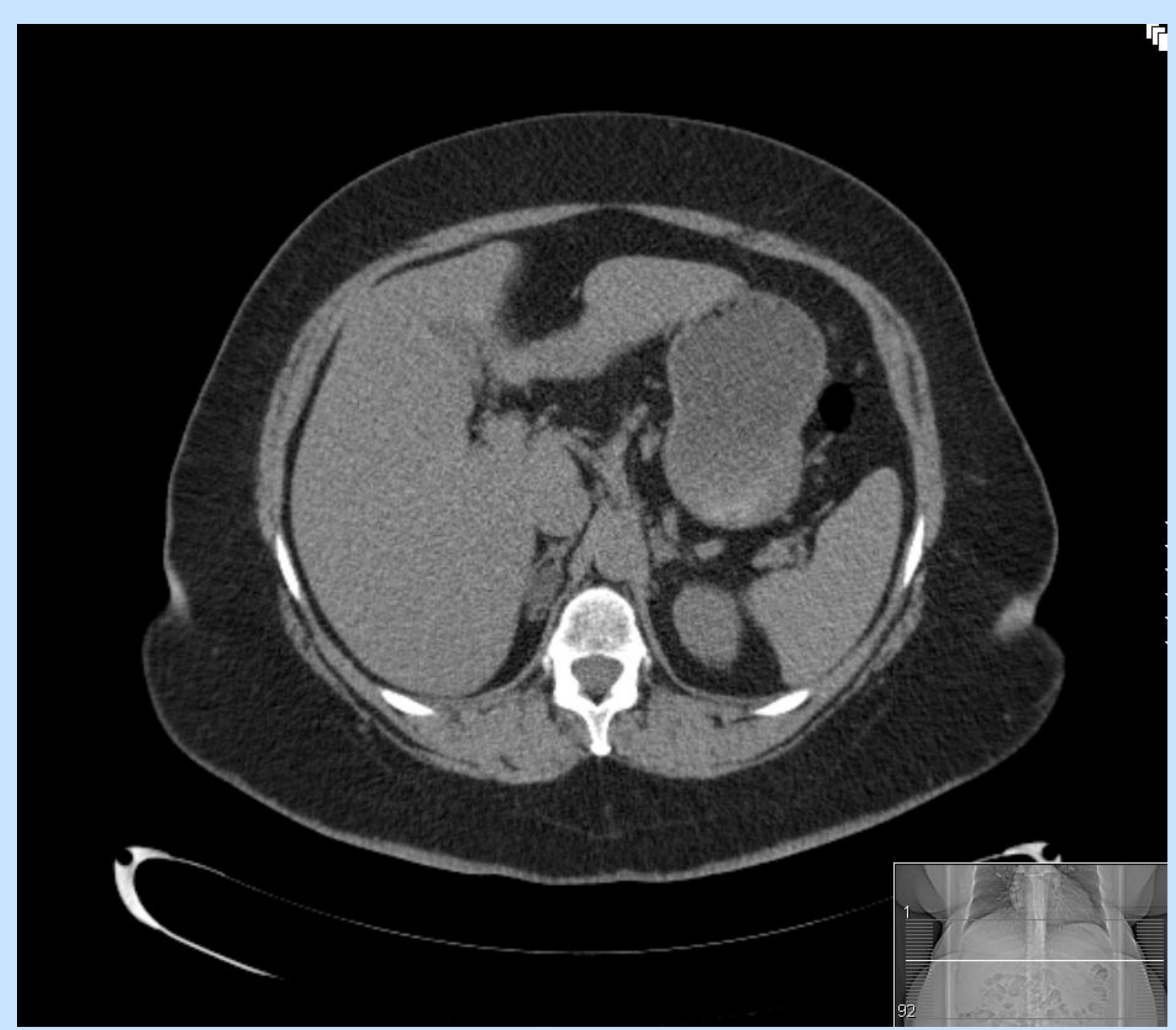

Fig.1- Unilateral adrenal adenoma

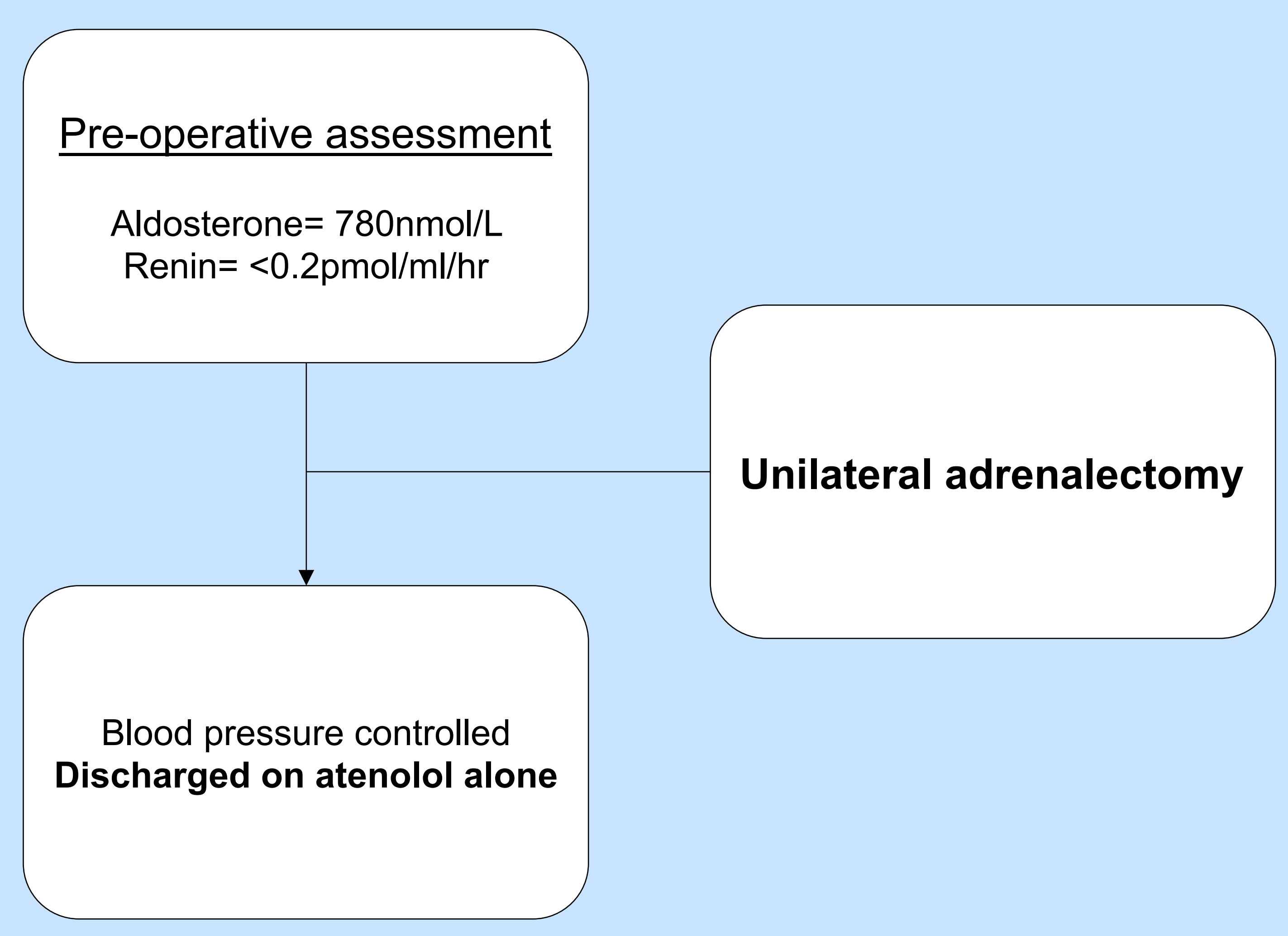

Fig.2- Perioperative management

\section{Postoperative presentations}

9 months after her adrenalectomy, the patient presented to Barnet Hospital with a variety of biochemical abnormalities consistent with hypoaldosteronism (fig. 3). These normalized following mineralocorticoid replacement. An attempt to reduce fludrocortisone dose in an outpatient setting resulted in a further reversible recurrence of both hyperkalaemia and acute kidney injury (fig. 4).

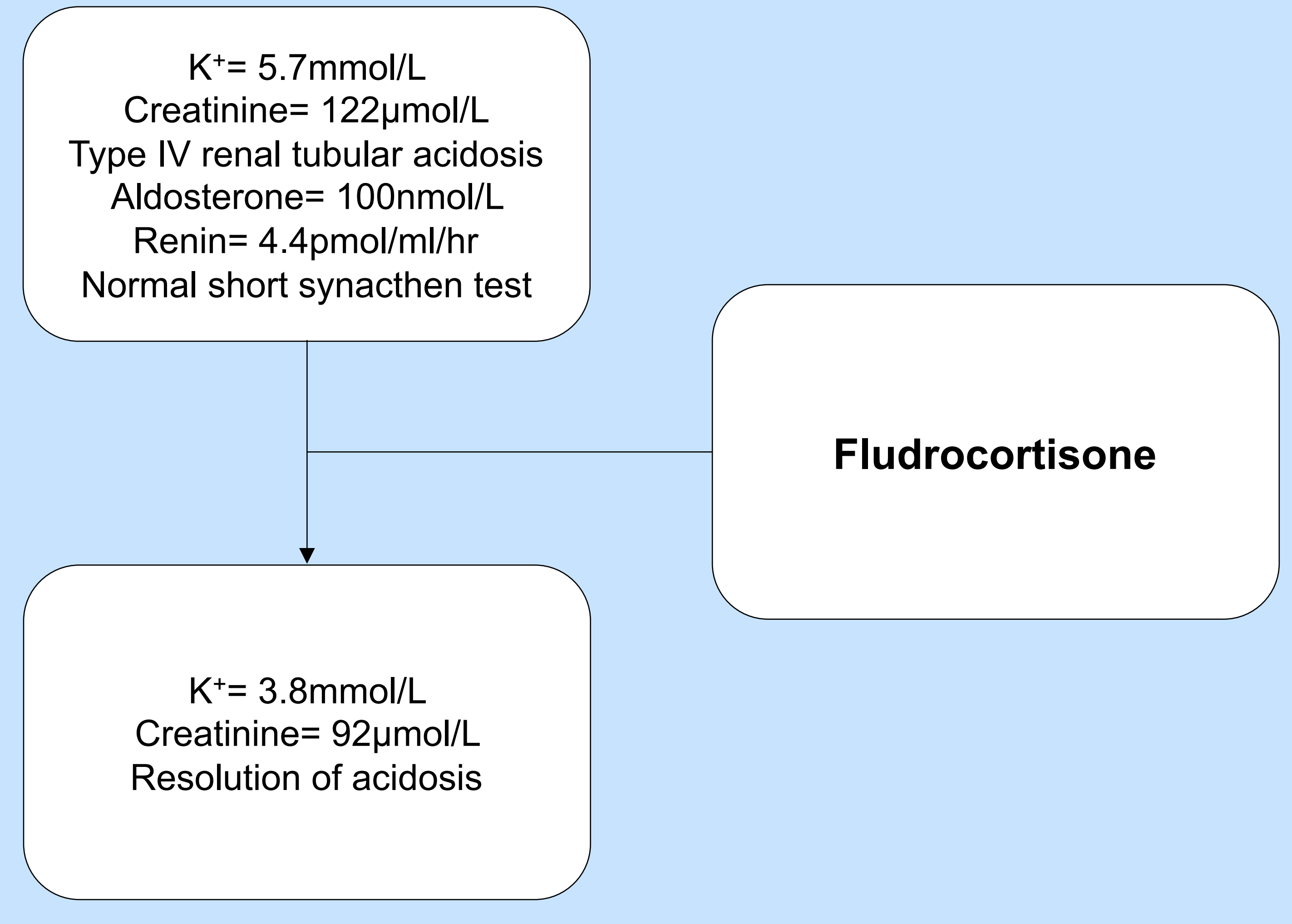

Fig. 3- Postoperative hypoaldosteronism

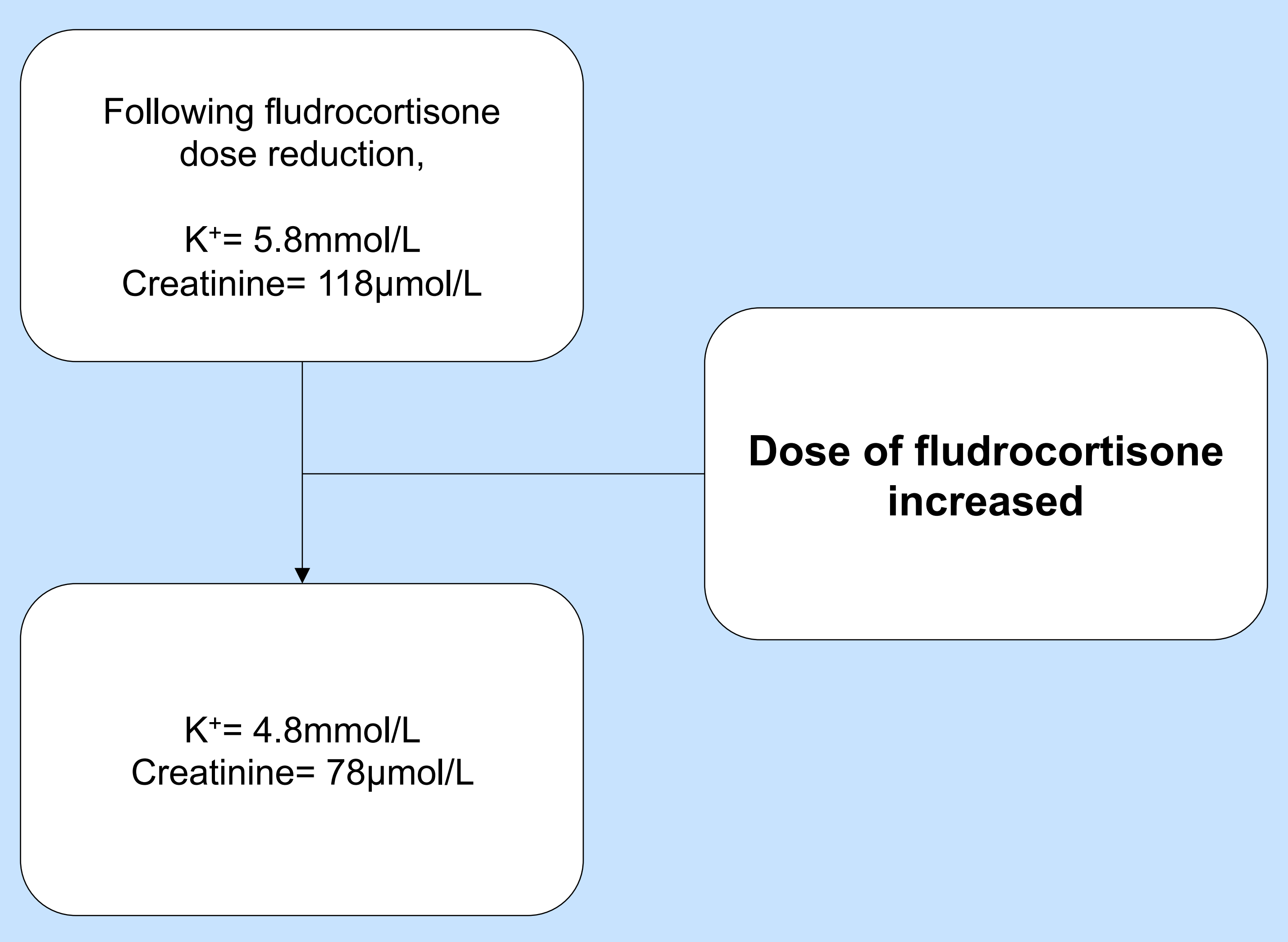

Fig. 4- Effects of altering mineralocorticoid replacement dose

\section{Conclusions}

- Post-operative hypoaldosteronism is well documented in cases of unilateral adrenalectomy for aldosteroneproducing adenomas.

- This may relate to a decrease in adrenal mass, or a transient suppression of the contralateral gland.

- However, it is rare for this to be prolonged, or severe. This may occur in up to $5 \%$ of cases, and responds to mineralocorticoid treatment. Our case illustrates the importance of follow-up in the post-operative period.

- B-blockade may have prevented adequate recovery of the patient's renin-aldosterone axis. Her atenolol has been now changed to amlodipine, and she awaits further follow up. 